\title{
Effect of anthocyanin-rich sour cherry extract on the level of IL-8 in LPS-induced endothelial cell
}

\author{
Attila Bíró - Arnold Markovics - László Stündl - Judit Remenyik \\ University of Debrecen, Faculty of Agricultural and Food Sciences and Environmental Management, Institute of Food Technology, H-4032 \\ Debrecen, Hungary \\ attila.biro88@gmail.com
}

\section{SUMMARY}

\begin{abstract}
The anthocyanin content of the Hungarian sour cherry is remarkable. Nutraceutical and pharmaceutical effects of the anthocyanins and their role in disease prevention have been studied extensively. Endothelial cells are involved in the pathogenesis of several inflammatory diseases. The objective of this work was to investigate pure sour cherry extract on human umbilical cord vein endothelial cells (HUVECs) as an inflammatory model. HUVECs were treated with $100 \mathrm{ng} \mathrm{mL}^{-1}$ lipopolysaccharide (LPS) and $50 \mu \mathrm{g} \mathrm{mL}^{-1}$ sour cherry extract or M199 medium as control. The optimal concentration range of the sour cherry extract was investigated and selected based on MTT assay measuring the conversion of the tetrazolium salt to formazan by mitochondrial dehydrogenases. The level of interleukine-8 (IL-8), a pro-inflammatory cytokine, was measured in Luminex MagPlex assay. LPS treatment significantly increased the secretion of IL-8. The pure sour cherry extract was able to attenuate this increment indicating the potent anti-inflammatory effect of pure sour cherry extract. Our results emphasize that pure sour cherry extract could reduce the LPS-induced inflammatory response thereby may improve endothelial dysfunction.
\end{abstract}

Keywords: sour cherry, anthocyanin, inflammation, endothelium

\section{INTRODUCTION}

Anthocyanins are water-soluble polyphenolic pigments that are responsible for the pigmentation of many foods, including fruits (sour cherry, blueberries, black plums, blackberries), vegetables (onion, red radish, purple cabbage), and grains (black rice, red rice, and black soybeans) (Khoo et al., 2017). Anthocyanins are a subgroup of flavonoids. Anthocyanins share a basic C-6 (A ring)-C-3 (C ring)-C-6 (B ring) carbon skeleton; it is also called the flavylium (2phenylchromenylium) ion, with a varying number of hydroxyl groups and sugars with different degrees of methylation. The stability of anthocyanin is dependent on $\mathrm{pH}$, light, temperature, and structure (Laleh et al., 2006).

The anthocyanin content of Hungarian sour cherry is outstanding, based on our preliminary investigations. They produce selective cyanidin glycosides, the main component being cyanidin-3-O-rutinoside (Homoki et al., 2016).

Several studies demonstrated that the anthocyanins can exert numerous beneficial physiological effects because of their anti-inflammatory, antioxidant, anti-obesity, anti-angiogenesis, anti-cancer, antidiabetes, anti-microbial, neuroprotection, and immunomodulation properties (Lila, 2004).

Many studies have focused on the defensive role of anthocyanins in inflammation. An in vitro study showed that the anthocyanin-rich bilberry extract is able to attenuate the IFN- $\alpha$-induced overexpression of MCP-1, IL-6, and TNF- $\alpha$ in human monocytic THP-1 cells. (Roth et al., 2014). Another in vitro study emphasized the protective effect of purified sour cherry anthocyanin extract on cytokine-induced inflammatory caco-2 monolayers (Nguyen et al., 2018).

In the present study, the effect of sour cherry anthocyanin extract was investigated in an inflammatory model. Because human umbilical cord vein endothelial cells (HUVECs) have been extensively used in studies of the physiology and pathophysiology of various diseases, including the inflammatory process (Medina-Leyte et al., 2020), we applied HUVECs as an inflammatory model. The inflammatory response was induced by lipopolysaccharide (LPS), an endotoxin from the outer membrane of bacteria, which is routinely used to trigger inflammation.

\section{MATERIALS AND METHODS}

\section{Preparation of Hungarian sour cherry (Prunus cerassus)}

Extraction and purification of sour cherry have been performed as described previously (Nemes et al., 2018). The analytical characterization and determination of flavonoids and proanthocyanidins was carried out using by UHPLC. The investigated compounds were separated using by Phenomenex Kinetex column $(2.6 \mu$, XB.C18, 100 X $4.6 \mathrm{~mm})$ and gradient elution. Methanol and $3 \%$ Formic acid in water was used as aluent. Flow rate was $0.7 \mathrm{~mL} \mathrm{~min}^{-1}$. UV-vis detection was used at $535 \mathrm{~nm}$ wavelength. Hungarian sour cherry cultivar, "Újfehértöi fürtös", was applied in our in vitro experiments

\section{Cell culture conditions}

The human umbilical vein endothelial cells (HUVECs) were isolated from human umbilical cords. Umbilical cords were obtained from the Department of Obstetrics and Gynaecology, Clinical Centre, University of Debrecen, Debrecen, Hungary. The HUVECs were isolated using by enzymatic digestion and maintained according to the method previously described (Biro et al., 2020). In our experiments, the M199 medium was used as a control. $100 \mathrm{ng} \mathrm{mL}^{-1}$ lipopolysaccharide (LPS) 
was used in cell culture to generate an inflammatory model.

\section{Determination of cellular viability}

The viability of the cells was determined by MTT (3-[4,5-dimethylthiazol-2-yl]-2,5 diphenyltetrazolium bromide) assay measuring the conversion of the tetrazolium salt to formazan by mitochondrial dehydrogenases. Cells were seeded to 96 -well plates at a density of 20000 cells per well in quadruplicates and were treated with anthocyanin extract of different concentrations (1, 5, 10, 50, 100, 500, $1000 \mu \mathrm{g} \mathrm{mL}^{-1}$ ) and without anthocyanin extract (control group) for 24 hours. The medium was removed, and the cells were then incubated with MTT reagent for 3 hours; subsequently, the formazan crystals were dissolved in $100 \mu \mathrm{L}$ solubilizing solution. The concentration of formazan crystals was determined colorimetrically at $465 \mathrm{~nm}$ by using Clariostar microplate reader (BMG Labtech, Ortenberg, Germany). Cell viability at different anthocyanin extract concentrations was expressed relative to $100 \%$ of the control group.

\section{Evaluation of the level of IL-8 on Luminex MagPlex assay8}

HUVECs were seeded into a 6-well plate $(500000$ cells/well) and were treated with M199 Medium as control and $100 \mathrm{ng} \mathrm{mL} \mathrm{mL}^{-1}$ LPS with or without $50 \mu \mathrm{g} \mathrm{mL}-1$ anthocyanin extract for 24 hours. The supernatant was collected and centrifuged for $10 \mathrm{~min}$ $10000 \mathrm{~min}^{-1}$ and stored at $-80{ }^{\circ} \mathrm{C}$. The levels of IL-8 were assessed using by MILLIPLEX MAP Human cytokine/chemokine Magnetic Bead Panel (HCYTOMAG-60K-09, EMD Millipore Corp., Billerica, MA, USA).

\section{Statistical analysis}

For multiple comparisons, results were analyzed by ANOVA followed by modified t-test for repeated measures according to Bonferroni's method. Data were presented as mean \pm SEM. Differences were considered statistically significant when $\mathrm{p}<0.05$.

\section{RESULTS AND DISCUSSION}

Main compounds of the purified sour cherry extract Based on our previous study (Nemes et al., 2018), the main anthocyanin components of the sour cherry were found as cyanidin-3-o-rutinoside, cyanidin-3-oglucoside, and cyanidin-3-o-glucosyl-rutinoside (Figure 1).

Figure 1: UHPLC chromatogram of sour cherry extract

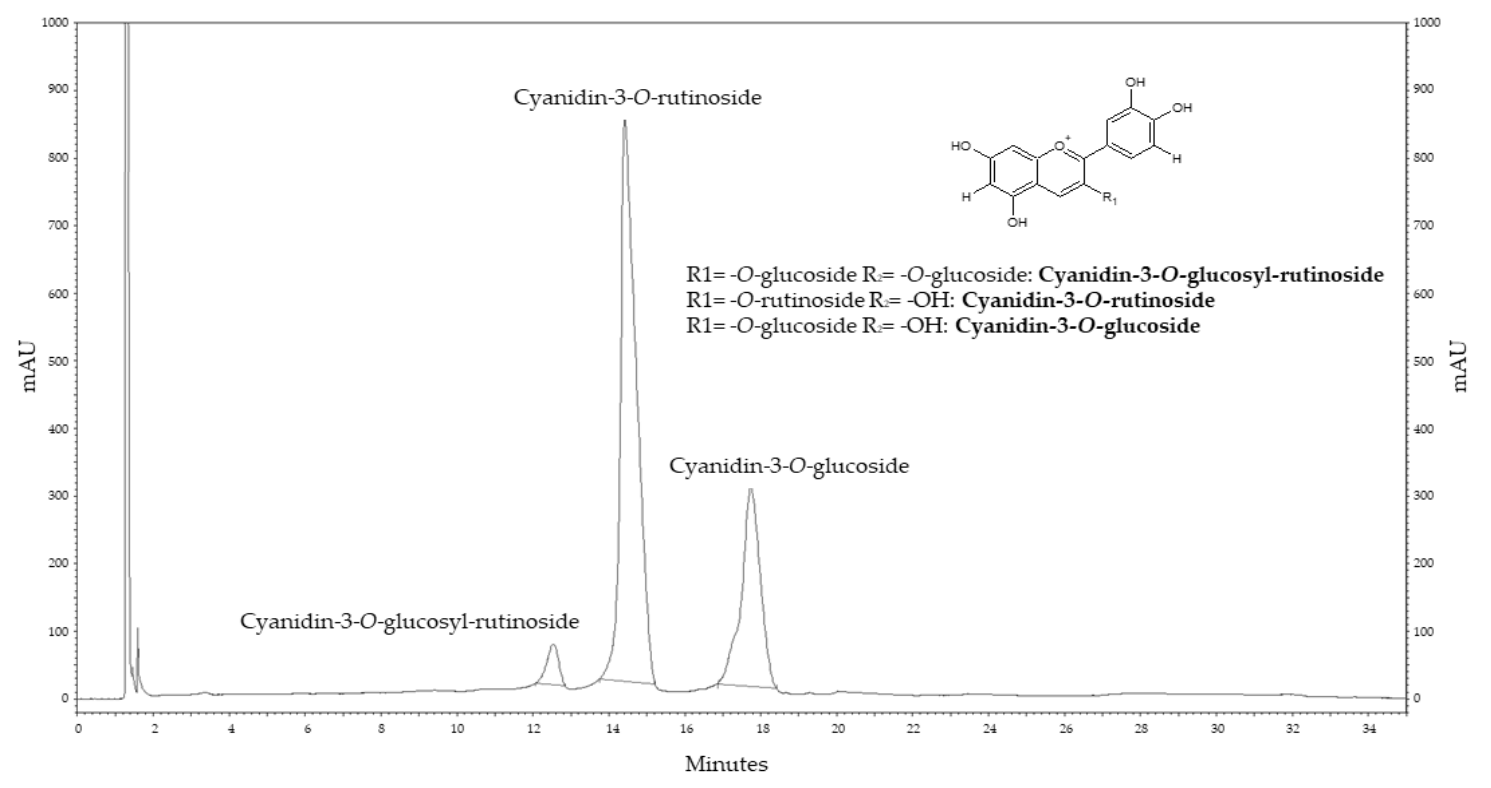

The optimal anthocyanin containing sour cherry extract concentration was determined by using MTTassay. We found that the high anthocyanin containing extract did not decrease the viability of HUVECs up to $500 \mu \mathrm{g} \mathrm{mL} \mathrm{m}^{-1}$ (Figure 2). These data suggest that relatively high concentrations of the anthocyanin containing extract can be applied without the possibility of cytotoxic effects. Moreover, we assessed that the highest anthocyanin containing extract concentration that significantly reduced the cell viability of HUVECs was $1000 \mu \mathrm{g} \mathrm{mL}^{-1}$ Given that the maximum of the main component (cyanidin 3-orutinoside) of the anthocyanin extract in blood is close to $50 \mu \mathrm{g} \mathrm{mL}^{-1}$ (Kay et al., 2017), that concentration was selected for further investigations. 
Figure 2: MTT-assays. The viability of HUVECs, was monitored followed up to 24 hours. The remaining reduction capacity of the cells were expressed in the percentage of the control samples. Data are expressed as the mean \pm SEM of four individual experiments. *means a significant difference between the data sets $\mathbf{p}<0.05$

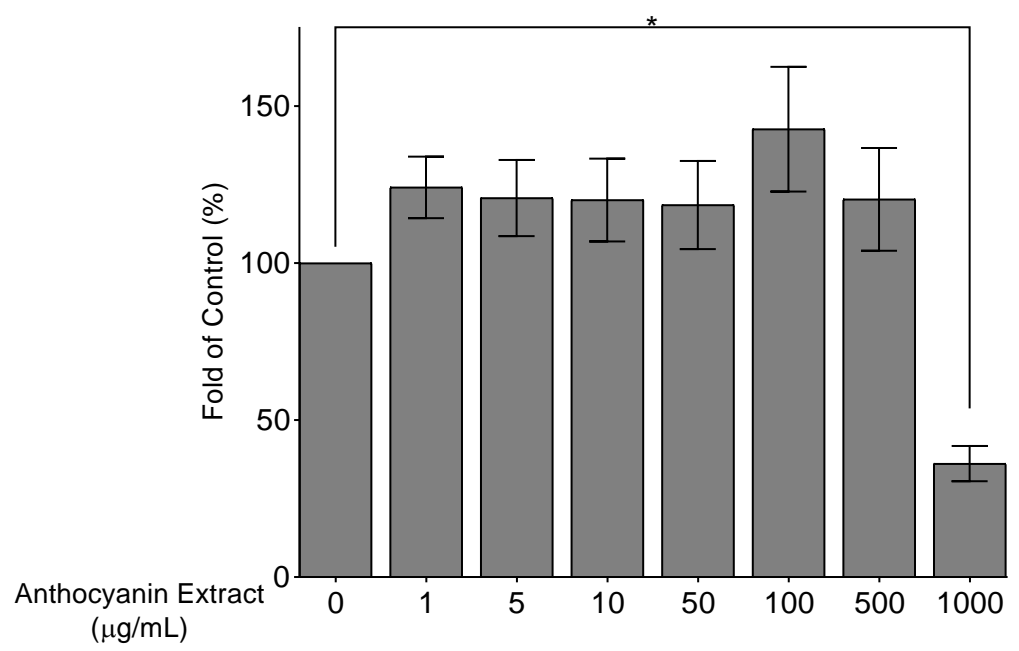

Numerous studies have demonstrated the antiinflammatory effects of anthocyanins (Vendrame et al., 2015) and IL-8 is one of the primary mediators of the inflammatory response. It plays a crucial role in the recruitment of the immune cells, primarily neutrophils, to the site of inflammation (Selders et al., 2017). We investigated the effect of the anthocyanins-rich sour cherry extract on the level of IL-8 increased due to the LPS-induced inflammatory response. As expected, administration of LPS significantly increased the level of IL-8. Anthocyanin extract was able to reduce this increment (Figure 3), indicating the significant antiinflammatory effect of our anthocyanin containing sour cherry extract.

Figure 3: After LPS-treatment, the level of IL-8 increased significantly compared to the control indicated by $*(P<0.05)$ * $*(P<0.005)$ indicates the significant changes in level of IL-8 in LPS-induced inflammation and the anthocyanin extract treatment compared to the level of IL-8 in LPS-induced inflammation without anthocyanin extract. Data are expressed as the mean \pm SEM of three individual experiments

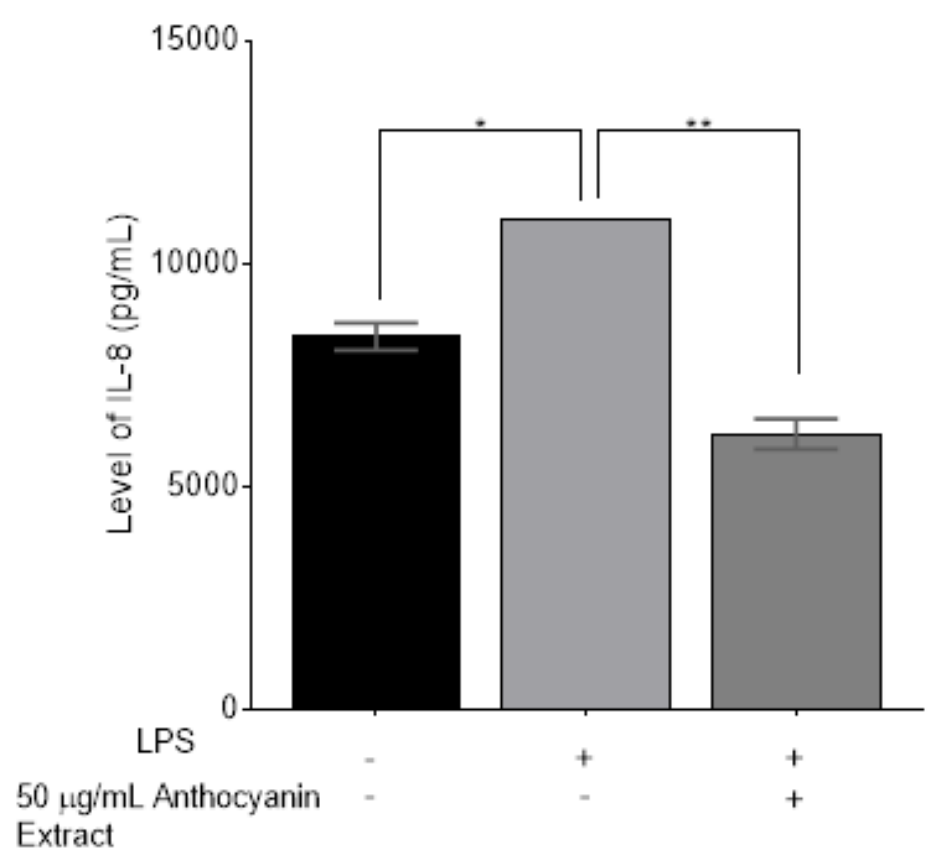




\section{CONCLUSIONS}

Plants produce several chemically-highly diverse secondary metabolites, which may be suitable for exerting positive effects on human diseases (Ncube et al., 2015). The object of this study was to investigate the effect of the anthocyanin-rich sour cherry extract on LPS-induced inflammatory response in HUVECs. Firstly, the main compounds of purified sour cherry extract were determined by UHPLC liquid chromatography. Subsequently, the non-cytotoxic concentration of anthocyanin extract was determined by using MTT-assay. Finally, we investigated the effect of anthocyanin extract on the level of IL-8, a proinflammatory cytokine, in LPS-induced HUVECs. A recent study reported the Protective effect of pure sour cherry anthocyanin extract on cytokine-induced inflammatory caco-2 monolayers (Nguyen et al., 2018). The authors showed that the pure sour cherry anthocyanin extract was able to decrease the TNF- $\alpha$ induced increased release of IL-8. In accordance with it, the anthocyanin-rich sour cherry extract significantly decreased the level of this pro-inflammatory cytokines. Several studies have demonstrated that anthocyanins can inhibit the cyclooxygenase-2 (COX-2) enzyme, thereby exerting an anti-inflammatory effect (Seeram et al., 2001). We hypothesize that the decrease in IL- 8 by the anthocyanin-rich sour cherry extract is due to the inhibition of COX-2 enzyme by the anthocyanins. However, further studies are needed to elucidate the mechanisms of action of anthocyanin extract. Our results suggest that anthocyanin extract may exert antiinflammatory effect and have therapeutic potential in inflammation-caused endothelial dysfunction.

\section{REFERENCES}

Biró, A.-Markovics, A.-Fazekas, É.M.-Fidler, G.-Szalóki, G.Paholcsek, M.-Lukács, J.-Stündl, L.-Remenyik, J. (2020): Allithiamine Alleviates Hyperglycaemia-Induced Endothelial Dysfunction. Nutirents 12(6), 1690.

Homoki, R.J.-Nemes, A.-Fazekas, E.-Gyémánt, Gy.-Balogh, P.Gál, F.-Al-Asri, J.-Mortier, J.-Wolber, G.-Babinszky, L.Remenyik J. (2016): Anthocyanin Composition, Antioxidant Efficiency, and $\alpha$-amylase Inhibitor Activity of Different Hungarian Sour Cherry Varieties (Prunus Cerasus L.). Food Chemistry 1;194:222-9.

Kay, D.C.-Pereira-Caro, G.-Ludwig, A.I.-Clifford, N.M.-Crozier, A. (2017): Anthocyanins and Flavanones Are More Bioavailable Than Previously Perceived: A Review of Recent Evidence. Annual Review of Food Science and Technology 8:155-180.

Khoo, E.H.-Azlan, A.-Tang, T.S.-Lim, M.S. (2017) Anthocyanidins and anthocyanins: colored pigments as food, pharmaceutical ingredients, and the potential health benefits. Food \& Nutrition Research. 61(1): 1361779.

Laleh, G.H.-Frydoonfar, H.-Heidary, R.-Jameei, R.-Zare, S. (2006): The Effect of Light, Temperature, $\mathrm{pH}$ and Species on Stability of Anthocyanin Pigments in Four Berberis Species. Pakistan Journal of Nutrition 5(1) 90-92.

Lila, A.M. (2004): Anthocyanins and Human Health: An In Vitro Investigative Approach. Journal of Biomedicine and Biotechnology 2004(5): 306-313.

Medina-Leyte J.D.-Domínguez-Pérez, M.-Mercado, I.-VillarrealMolina, T.M.-Jacobo-Albavera, L. (2020): Use of Human Umbilical Vein Endothelial Cells (HUVEC) as a Model to Study Cardiovascular Disease: A Review. Applied Science 10(3), 938.

Nguyen, P.L.T.-Fenyvesi, F.-Remenyik, J.-Homoki, R.J.-Gogolák, P.-Bácskay, I.-Fehér, P.-Ujhelyi, Z.-Vasvári, G.-Vecsernyés, M.-Judit Váradi, J. (2018): Protective Effect of Pure Sour Cherry Anthocyanin Extract on Cytokine-Induced Inflammatory Caco2 Monolayers. Nutrients 10(7): 861
Ncube, B.-Van Staden, J. (2015): Tilting Plant Metabolism for Improved Metabolite Biosynthesis and Enhanced Human Benefit. Molecules 20(7): 12698-12731.

Nemes, A.-Szöllősi, E.-Stündl, L.-Biró, A.-Homoki, R.J.-Szarvas, M.M.-Balogh, P.-Cziáky, Z.-Remenyik, J. (2018): Determination of Flavonoid and Proanthocyanidin Profile of Hungarian Sour Cherry. Molecules 11;23(12):3278.

Nguyen, P.L.T.-Fenyvesi, F.-Remenyik, J.-Homoki, R.J.-Gogolák, P.-Bácskay, I.-Fehér, P.-Ujhelyi, Z.-Vasvári, G.-Vecsernyés, M.-Judit Váradi, J. (2018): Protective Effect of Pure Sour Cherry Anthocyanin Extract on Cytokine-Induced Inflammatory Caco2 Monolayers. Nutrients 10(7): 861.

Roth, S.-Spalinger, R.M.-Müller, I.-Lang, S.-Rogler, G.-Scharl, M. (2014): Bilberry-derived Anthocyanins Prevent IFN- $\gamma$-induced Pro-Inflammatory Signalling and Cytokine Secretion in Human THP-1 Monocytic Cells. Digestion 90(3):179-89

Seeram, N.P.-Momin, R.A.-Nair, M.G.-Bourquin, L.D. (2001): Cyclooxygenase inhibitory and antioxidant cyanidin glycosides in cherries and berries. Phytomedicine 8(5):362-9.

Selders, S.G.-Fetz, E.A.-Radic, Z.M.-Bowlin, L.G. (2017): An overview of the role of neutrophils in innate immunity, inflammation and host-biomaterial integration. Regenerative Biomaterials 4(1): 55-68.

Vendrame, S.-Klimis-Zacas, D. (2015): Anti-inflammatory Effect of Anthocyanins via Modulation of Nuclear factor- $\mathrm{\kappa} B$ and Mitogen-Activated Protein Kinase Signaling Cascades. Nutrition Reviews 73:6 348-358. 